\title{
Constructing a Water-Energy-Food Efficiency Coupling Model from the Perspective of Land Use
}

\author{
Qingmu SU ${ }^{\mathrm{a}}$ and Kaida CHEN ${ }^{\mathrm{a}, \mathrm{b}, 1}$ \\ a Department of Urban Planning, National Cheng Kung University, Taiwan 70101, \\ China \\ ${ }^{\mathrm{b}}$ Landscape Architecture, School of Landscape Architecture, Fujian Agriculture and \\ Forestry University, Fujian 350002, China
}

\begin{abstract}
Water, energy and food are basic human needs. However, there is a competitive relationship among these three types of resources regarding land resources. How to study the coupling and coordination relationship among waterenergy-food (W-E-F) from the perspective of land use has become a problem demanding prompt solution. In this study, parallel data envelopment analysis and coupling coordination degree (CCD) model were used to establish an efficiency coupling model of W-E-F. The model aims to explore the interrelationship among the three kinds of important resources as a whole and establish a synergistic collective solution. The results showed that the relationship among water, energy and food with a high CCD can improve the overall parallel efficiency. The results can provide a reference for the development of long-term coordination plan and action plan of departments to achieve Sustainable Development Goals.
\end{abstract}

Keywords. Land resources, water-energy-food, sustainable development

\section{Introduction}

Water, energy and food (W-E-F) are basic human needs, which are vital to human survival and social and economic development. However, population growth and rapid urbanization across the world have put tremendous pressure on limited W-E-F resources. By 2030, the demand for these resources is expected to increase by $40 \%$, $50 \%$, and $35 \%$ respectively [1]. In addition, the imbalanced regional distribution of Water, energy and food resources and the huge differences in development level have caused different degrees of impact on the efficiency of resource utilization. Therefore, the world today has realized the importance of the reasonable and effective utilization of W-E-F. Most of the previous researches focus on the relationship between energy and water, the relationship between food and water, and the relationship between energy and food [2]. Few studies have explored the relationship among these three systems. Besides, these three types of resources often rely on land use to be transformed into output. Therefore, how to study the relationship among W-E-F from

\footnotetext{
${ }^{1}$ Corresponding Author, Kaida CHEN, Landscape Architecture, School of Landscape Architecture, Fujian Agriculture and Forestry University, Fujian 350002, China; Email: kaidachenwhu@gmail.com.
} 
the perspective of land use has become an issue that needs to be solved for sustainable development.

There is a competitive relationship among the Energy, water and food sectors in terms of land resources. Land development and its resilience vary among regions due to different conditions, and the changes in land use are often irreversible [3]. Therefore, the utilization efficiency of W-E-F resources based on different types of land will determine whether the method of land development can be maintained.

In the current resource utilization model, obtaining sufficient Energy, water and food resources to meet the needs of urban development will become more challenging. In fact, the three subsystems of W-E-F are interdependent and highly related to each other [4]. In order to achieve the sustainable development of W-E-F resources, the sustainable management of these three types of basic resources needs to be transformed from a single management method to a unified measurement method [5]. In this study, parallel data envelopment analysis was adopted to improve the efficiency of resource management and avoid the inefficiency caused by the failure of the decision-making of a single sector [6]. Most importantly, this method can help us understand the resource utilization efficiency of the entire system and how to adjust resource utilization.

Combining the measurement of the efficiency of water, energy and food resources with land use is of great significance for effectively managing various sectors and promoting sustainable development. In this study, parallel DEA and the coupling coordination degree method were used to establish an efficiency coupling model of energy, water and food. The purposes are (1) to evaluate the interaction among the WE-F subsystems; (2) to assess the overall performance of the coupling system and to investigate the energy, water and food coupling in each county and city in Taiwan Province, so as to judge whether the W-E-F development method can achieve sustainable development.

\section{Methodology}

\subsection{Indicator Construction and Method}

Data envelopment analysis (DEA) can objectively evaluate the relative efficiency of decision-making units with multiple inputs and outputs of different units. However, the traditional DEA did not open the internal structure of the model, which would lead to the loss of related information [7]. The super-efficient parallel DEA model (The structure is shown in figure 1) used in this study can be used to evaluate the efficiency of each part of the water, energy and food systems and the overall efficiency [8]. 


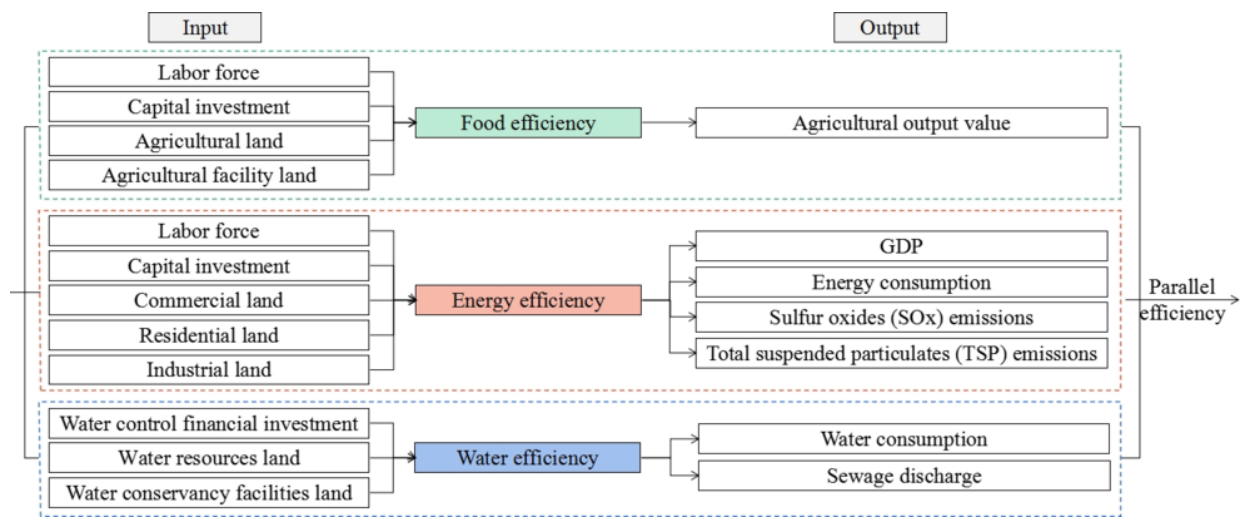

Figure 1. The efficiency indicators of food, energy and water and the structure of the parallel system.

In this paper, a weighted average efficiency formula was proposed to evaluate the overall utilization efficiency of food, energy and water. Suppose that the structure of the parallel system is shown in figure 1, then the parallel DEA model is as follows:

$$
\begin{aligned}
& \operatorname{Max} \theta_{0}= \phi_{1} \theta_{10}+\phi_{2} \theta_{20}+\phi_{3} \theta_{30} \\
& \text { s.t. } \quad \theta_{\mathrm{k} j}=\frac{\sum_{\mathrm{r}=1}^{s} \mu_{r} y_{\mathrm{r}_{0}}+\sum_{\mathrm{r}=1}^{s} \lambda_{r} \bar{z}_{r j_{0}}}{\sum_{i=1}^{m} v_{i} x_{i j}+\sum_{i=1}^{m} \varsigma_{i} \alpha_{k} g_{i j}} \leq 1, \\
& \alpha_{1}+\ldots+\alpha_{k}=1, \\
& \mu_{\mathrm{r}}, v_{i}, \lambda_{r}, \varsigma_{i}, \alpha_{k} \geq 0, \quad i=1,2 \ldots \mathrm{m}, j=1,2 \ldots n \text { and } \quad j \neq j_{0}, r=1,2 \ldots s, k=1,2,3
\end{aligned}
$$

where $\theta_{10}, \theta_{20}, \theta_{30}$ represent the utilization efficiency of water, energy and food resources respectively; $\phi_{1}, \phi_{2}, \phi_{3}$ represent the weight of the parallel subsystems respectively, that is, one-third of the weights in this paper. $\alpha_{1}, \alpha_{2}, \alpha_{3}$ denote the proportion of the land resources consumed by water, energy and food separately. By adjusting the ratio of $\alpha_{1}, \alpha_{2}, \alpha_{3}$, the overall efficiency of food, energy and water can be enhanced to promote more effective utilization of land resources.

Based on the efficiency of DEA, coupling degree and coupling coordination degree were adopted to quantify the interaction among W-E-F subsystems. The coupling degree emphasizes the intensity of the interaction among these systems, and the coupling coordination degree highlights the positive interaction among the systems [9].

Formula (2) shows the coupling degree among water, energy and food subsystems. The coupling degree was divided into four levels: non-coupling (0-0.25); antagonistic coupling (0.25-0.5); basic coupling (0.5-0.75); strong coupling (0.75-1).

$$
C=\left\{\frac{\theta_{1} \times \theta_{2} \times \theta_{3}}{\left(\frac{\theta_{1}+\theta_{2}+\theta_{3}}{3}\right)^{3}}\right\}^{\frac{1}{3}}
$$


Formula (3) shows the coupling coordination degree (CCD) among water, energy and food subsystems, involving four stages: low CCD stage (0-0.25); moderate CCD stage (0.25-0.5); high CCD stage (0.5-0.75); extreme CCD stage (above 0.75). In the $\mathrm{W}-\mathrm{E}-\mathrm{F}$ relationship, $\mathrm{a}=\mathrm{b}=\mathrm{c}=1 / 3$.

$$
D=\sqrt{C \times\left(a \theta_{1}+b \theta_{2}+c \theta_{3}\right)}
$$

\section{Results}

Table 1 shows the efficiency of the food, energy and water subsystems and the overall parallel efficiency in each county and city. The overall efficiency of food, energy and water in the counties and cities in Taiwan Province is quite different. The average efficiency of water is the lowest, only 0.51 , and the efficiency of energy utilization is the highest. Besides, except that Taipei City has a high overall efficiency, other cities do not have high efficiency of W-E-F. It can be seen from the last two columns of table 1 that the variation trend of the coupling degree and the CCD is relatively similar. On the whole, the coupling degree is greater than the coupling coordination degree, indicating that the internal interaction among W-E-F is stronger than the degree of coordination among these subsystems. The comparison of efficiency and coupling coordination degree showed that the W-E-F relationship with high CCD can improve the overall parallel efficiency.

Table 1. Parallel efficiency and coupling of water-energy-food.

\begin{tabular}{lllllll}
\hline & \multicolumn{7}{c}{ Food Energy } & Water Parallel efficiency Coupling degree Coupling coordination degree \\
\hline Taipei city & 0.03 & 3.82 & 0.06 & 1.30 & 0.14 & 0.42 \\
Kaohsiung city & 0.49 & 0.30 & 0.01 & 0.27 & 0.44 & 0.34 \\
New Taipei city & 0.09 & 0.45 & 0.02 & 0.19 & 0.46 & 0.29 \\
Yilan county & 0.46 & 0.37 & 0.55 & 0.46 & 0.99 & 0.67 \\
Taoyuan city & 0.15 & 0.29 & 0.04 & 0.16 & 0.73 & 0.34 \\
Hsinchu county & 1.15 & 1.03 & 0.51 & 0.90 & 0.94 & 0.92 \\
Miaoli county & 0.44 & 0.32 & 0.45 & 0.40 & 0.99 & 0.63 \\
Taichung city & 0.35 & 0.29 & 0.01 & 0.22 & 0.48 & 0.32 \\
Changhua county & 1.03 & 0.21 & 0.63 & 0.62 & 0.83 & 0.72 \\
Nantou county & 1.03 & 0.30 & 0.43 & 0.59 & 0.87 & 0.72 \\
Yunlin county & 1.21 & 0.17 & 0.45 & 0.61 & 0.75 & 0.67 \\
Chiayi county & 1.33 & 0.28 & 1.32 & 0.98 & 0.81 & 0.89 \\
Tainan city & 0.47 & 0.23 & 0.04 & 0.25 & 0.67 & 0.41 \\
Pingtung county & 1.45 & 0.24 & 0.18 & 0.62 & 0.63 & 0.63 \\
Taitung county & 1.02 & 2.91 & 1.80 & 1.91 & 0.91 & 1.32 \\
Hualien county & 0.45 & 0.48 & 0.49 & 0.47 & 1.00 & 0.69 \\
Keelung city & 3.13 & 1.42 & 0.53 & 1.69 & 0.79 & 1.16 \\
Hsinchu city & 0.05 & 1.01 & 0.19 & 0.42 & 0.52 & 0.47 \\
Chiayi city & 0.11 & 1.38 & 2.00 & 1.16 & 0.58 & 0.82 \\
Average & 0.76 & 0.82 & 0.51 & 0.70 & 0.71 & 0.65 \\
\hline & & & & & & \\
\hline
\end{tabular}




\section{Conclusion}

Due to the increasing social and economic stress, the demand for W-E-F has increased unprecedentedly and has exceeded the carrying capacity of land use. If the relationship between land use and W-E-F is ignored, it may have a negative impact on the sustainable development goals. In this study, a parallel DEA model was proposed from the perspective of land use to measure the coupling efficiency of food, energy and water. This model not only describes the efficiency of each part of food, energy and water, but also measures the overall efficiency of W-E-F. Investigating the resource utilization efficiency and overall coupling of W-E-F is not only important for sustainable development goals (SDG 2: Eliminate hunger, SDG 6: Enjoy water and sanitation and its sustainable management, SDG 7: Affordable modern energy) [10], but also it is essential for achieving the integration of the three resource systems and sectors. In short, only when the food, energy and water subsystems are in a coupled and coordinated state can the parallel connection of W-E-F achieve high efficiency. The research results can provide a reference for the development of long-term coordination plan and action plan of departments.

\section{Reference}

[1] Zhang P, Zhang L, Chang Y, Xu M, Hao Y, Liang S, Liu G, Yang Z and Wang C 2019 Food-energywater (FEW) nexus for urban sustainability: A comprehensive review Resources, Conservation and Recycling 142 215-224.

[2] Deng H, Wang C, Cai W, Liu Y and Zhang L 2020 Managing the water-energy-food nexus in China by adjusting critical final demands and supply chains: An input-output analysis Science of The Total Environment 720137635.

[3] Ogbolumani O and Nwulu N 2021 Multi-objective optimisation of constrained food-energy-waternexus systems for sustainable resource allocation Sustainable Energy Technologies and Assessments $\mathbf{4 4}$ 100967.

[4] Li P and Ma H 2020 Evaluating the environmental impacts of the water-energy-food nexus with a lifecycle approach Resources, Conservation and Recycling 157104789.

[5] Arthur M, Liu G, Hao Y, Zhang L, Liang S, Asamoah E F and Lombardi G V 2019 Urban food-energywater nexus indicators: A review Resources, Conservation and Recycling 151104481.

[6] Su Q and Chen X 2021 Efficiency analysis of metacoupling of water transfer based on the parallel data envelopment analysis model: A case of the South-North water transfer project-middle route in China Journal of Cleaner Production 313127952.

[7] Su Q 2020 Long-term flood risk assessment of watersheds under climate change based on the game cross-efficiency DEA Natural Hazards 104 2213-2237.

[8] Sun C, Yan X and Zhao L 2021 Coupling efficiency measurement and spatial correlation characteristic of water-energy-food nexus in China Resources, Conservation and Recycling 164105151.

[9] Han D, Yu D and Cao Q 2020 Assessment on the features of coupling interaction of the food-energywater nexus in China Journal of Cleaner Production 249119379.

[10] Wang K, Liu J, Xia J, Wang Z, Meng Y, Chen H, Mao G and Ye B 2021 Understanding the impacts of climate change and socio-economic development through food-energy-water nexus: A case study of Mekong river delta Resources, Conservation and Recycling 167105390. 